\title{
Guest Editorial Special Section on Sensor Applications
}

$\mathbf{T}$ HE SENSOR applications are one of the primary research topics in the worldwide frontiers for technologies, such as smart city, better society, energy, health, environment, and industry. The IEEE Sensor Applications Symposium (SAS) has been the leading forum to present novel approaches and emerging applications in sensor technology, including biosensors and arrays, smart sensors and standards, sensor networking, microelectromechanical systems and nanosensors, virtual sensors, homeland security, multisensor data fusion, nondestructive evaluation and remote sensing, integrated system health management (ISHM), and commercial development. This special section on the seventh edition, SAS 2012, includes six papers from the symposium that illustrate the diversity of sensor applications that we have seen during the conference.

The call for papers at SAS 2012 resulted in more than 100 submitted papers, which has been at an increasing pace compared with previous years. The contributions were reviewed by an international technical program committee. To ensure high quality, each paper was evaluated by three independent reviewers. As a result, over 50 papers were accepted for the symposium presentation and publication. From the submitted papers to the Transactions, six contributions were significantly extended in order to qualify for publication in this special section of the IEEE TRANSACTIONS ON INSTRUMENTATION and MEASUREMENT. Many of the papers deal with outstanding research topics proposing innovative approaches, and I recommend to have a look at the conference program and the conference proceedings. The reviewers were instructed not only to comment on the technical excellence of the content but also on the significant technical extension, as compared with the original symposium paper.
The following is a brief synopsis of the papers in this SAS 2012 Special Section. The paper by T. Addabbo et al. is on one of the main concerns in motion control, i.e., the measurement of the angular velocity variations of a rotating shaft, where the authors propose an interesting measurement method based on the zero-crossing demodulation technique of FM signals. Sensors for industrial applications are also the topic of the paper by A. Bernieri et al., investigating eddy current testing to detect the presence of thin defects (cracks) in conductive materials. The paper by L. Fanucci et al. proposes an innovative home care model in order to support in an integrated and coordinated fashion the whole process of the patient treatment, connecting in-hospital care with out-of-hospital follow up by means of remote sensing of vital signs. The paper by E. Marenzi et al. proposes a suitable capacitive sensor matrix to detect the level of comfort and discomfort during long periods of sitting. Submicrosecond synchronization per hop in wireless sensor networks is the result of the paper by M. Akhlaq et al., due to a recursive time synchronization protocol, and an orientation tracking system based on a double-stage Kalman filter in a 9-D inertial measurement unit is developed in the paper by S. Sabatelli et al.

I would like to thank the authors for their submissions and their effort in doing revisions, as suggested by the reviewers, and the final excellent quality of their papers. I also would like to thank the reviewers and the Chief Editor for their meticulous reviews and emphasis on detail to maintain the high-quality standard of the Transactions. Special thanks go to Reta Wehmeier and Cam Ingelin for their help and patience in administering the entire publication process.

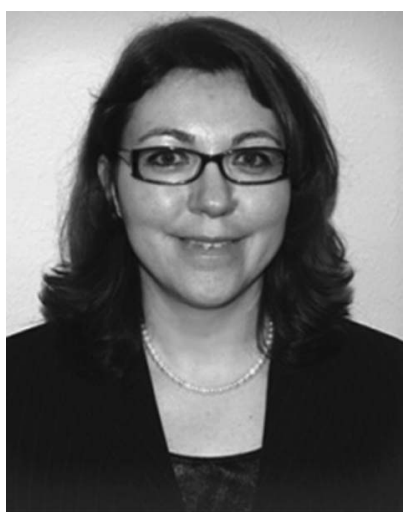

Deniz Gurkan received the B.S. and M.S. degrees from Bilkent University, Ankara, Turkey, in 1996 and 1998, respectively, and the Ph.D. degree in 2003 from the University of Southern California, Los Angeles, CA, USA, all in electrical engineering. During her Ph.D. studies, she has demonstrated optical networking functions with all-optical setups.

In 2004, she was with the Applied Mathematics Program, Claremont Graduate University, Claremont, CA, USA, and with the Department of Electrical Engineering, California State University at Long Beach, Long Beach, CA, USA, before joining the Department of Engineering Technology, University of Houston, Houston, TX, USA, where she is currently a Professor. She is the author or coauthor of more than 50 peer-reviewed technical papers in her field of research. She is currently involved in building research network test beds and interoperable distributed networking systems. Her current research interests include computer networks, sensor network standard development, and measurement systems.

Dr. Gurkan serves on the Steering Committee and the Technical Program Committee of the IEEE Sensor Applications Symposium and will chair the 2013 IEEE Sensors Applications Symposium in Galveston, TX. She is the Associate Editor for the IEEE TRANSACTIONS ON INSTRUMENTATION and MEASUREMENT. 


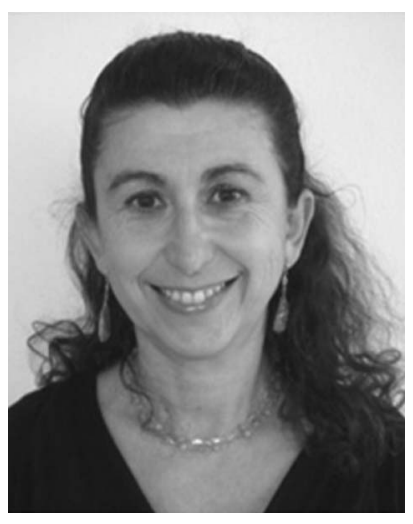

Alessandra Flammini was born in Brescia in 1960. She received the Laurea degree in physics with the University of Rome, Rome, Italy, in 1985.

From 1985 to 1992, she was employed in Ansaldo Industria, Milan, Italy, and in 1990, she became the Manager of the Digital Controls Unit, Ansaldo Industria. In 1995, she joined the University of Brescia, Brescia, Italy, and in 2002, she became an Associate Professor of electronic measurements and became responsible for the Electronics Laboratory. In 2004, she realized the National Competence Center of Profibus Network Italia. She has been responsible for the Brescia unit in two projects (PRIN2005 and PRIN2008). She is the coauthor of more than 150 international papers (scientific journals, patents and conference proceedings). Her research interests include smart sensors and sensor signal processing, real-time Ethernet, and wireless sensor networks based on several technologies; software-defined radio for real-time applications; smart grids; smart meters; and synchronization.

Ms. Flammini has been the General Co-chair of international IEEE conferences (2009 International IEEE Symposium on Precision Clock Synchronization for Measurement, Control and Communication (ISPCS) and 2012 IEEE Sensors Applications Symposium). She has been elected for a four-year term (2013-2016) in the Administrative Committee of the IEEE Instrumentation and Measurement Society. Some papers that she coauthored have been awarded (e.g., in the 2008 IEEE International Conference on Emerging Technologies and Factory Automation and in the 2010 ISPCS). 